\title{
BlueSpace: Personalizing Workspace through Awareness and Adaptability
}

\author{
Jennifer Lai, Anthony Levas, Paul Chou, Claudio Pinhanez, Marisa Viveros \\ IBM T.J. Watson Research Center, 19 Skyline Drive, Hawthorne, NY, 10532 \\ jlai, levas, pchou, pinhanez, viveros@us.ibm.com
}

\begin{abstract}
This paper reports on research in workplace issues encountered by knowledge workers in cubicle environments, and on BlueSpace, a prototype workspace with the goal of addressing workers' critical needs for privacy, concentration and personalization. To inform the design process, more than fifty on-site interviews with knowledge workers were conducted at six companies ranging from dot.com startups to Fortune 100 corporations. Several common requirements emerged including the need for a sense of control of one's workspace, the ability to create privacy on-demand to improve concentration and minimize unwanted interruptions, as well as in-place support for dyadic interactions. Many other common workplace complaints (e.g., too hot, too cold, too noisy) were found to be derivative of the major requirements for individual control and privacy.
\end{abstract}

\section{Introduction}

Many companies in the United States are looking to create flexible workspaces in order to support collaborative work and to respond quickly to dynamic changes in the business environment. Adaptable workspaces have the potential to speed information flow, facilitate spontaneous team interactions, and support business processes (Bauman \& Arens, 1996). A growing trend is the use of open-plan offices where workers inhabit semi-walled areas commonly known as cubicles. As offices evolve from rigid, closed environments into flexible, open spaces, individual comfort and privacy become of greater concern. The corporate goal of achieving economies of scale and space through the installation of uniform cubicles can occasionally overshadow employees' needs for individuality and quiet concentration. In addition to installing cubicles, many companies are using strategies that include unassigned space (sometimes referred to as hoteling) and mobility centers (Bauman, Arens, 1996) in an attempt to increase the efficiency of space utilization. These strategies allocate space to employees based on their needs for a given day or week, rather than dedicating a fixed office to every employee. 
The lack of full-sized, fixed walls contributes to increased noise levels on the floor, lack of auditory privacy, and to difficulties in personalizing the temperature in the workspace. Studies have shown a measurable impact on productivity associated with environmental factors such as temperature settings (Fisk and Rosenfeld 1997, Mendell 1993), as well as number of interruptions, lack of privacy and noise levels (Budd, 2001), (DeMarco \& Lister, 1999).

Cubicles are often viewed as an unhappy compromise between radical collocation (Teasley, Covi, Krishnan, Olson, 2000) and private offices in that they don't provide the benefits of either of these two types of workspaces. In a field study of six radically collocated software development teams, Teasley et al. (2000) found a doubling of productivity due to increased coordination of work activities, learning and persistence of work artifacts. On the opposite end of the spectrum, privacy and quiet were shown to have a beneficial impact in a study by DeMarco and Lister (1999) that examined a series of performance measures during "coding war games" in which 600 developers from 92 companies participated over the course of 2 years. High performance metrics from the coding games were associated with workspace environments that were substantially different between workers in the the top quartile, (those who did the exercise most rapidly and effectively) and those in the bottom quartile. DeMarco and Lister noted that the top perfomers' space is "quieter, more private, better protected from interruption, and there is more of it."

Given the current array of communication tools, knowledge workers can often work from home or some other remote location, however coming to the office provides workers with the added value of interacting with colleagues. O'Conaill and Frolich (1995) shadowed two mobile professionals, and analyzed 125 naturally occurring interruptions in 29 hours of video. They found that in $64 \%$ of the interruptions, the recipient of the 
interruption received some benefit. For $43.2 \%$ of the interruptions, both parties profited from the interruption. Since part of the value that knowledge workers provide to their employers is by sharing their knowledge with others, it stands to reason that there is a negative impact when these workers telecommute and are unavailable for informal, and serendipitous interactions - what Whittaker, Frohlich and Daly-Jones (1994) refer to as lightweight interactions. These informal interactions are defined as "generally impromptu, brief, context-rich and dyadic" (Nardi, Whittaker and Bradner, 2000) and support joint problem solving, coordination, social bonding and social learning. Whittaker et. al (1994) discuss the importance of lightweight or informal interactions, as shown by research demonstrating that people who are physically collocated are more likely to communicate frequently, researchers are more likely to be familiar with and respect the work of colleagues that sit near to them, and that opportunistic conversations are vital to the planning and definitional phases of projects. Thus, a key issue in workplace productivity for knowledge workers is to ensure a balance between their need to "cordon off" time for quiet uninterrupted work and the benefits of having them present in the workplace, accessible to others, and working in an environment that is supportive of lightweight and serendipitous interactions.

Our research was motivated by an interest in creating workspace solutions which could be reproduced throughout office buildings with the same ease as cubicles, but that addresses many of the common complaints associated with cubicles. The goal of the solution was to combine existing technologies (e.g., flat panel screens, sensors) along with technologies which are just emerging from the research evironment in a novel way to resolve knowledge workers' problems. In order to obtain first-hand knowledge of the current workplace issues and to inform the design process, we spent time with knowledge workers to understand how they feel about working in cubicles. This article first reports on the findings from these sessions, then 
describes a prototype workspace solution that we built to address some of the most common issues raised. In the last section we discuss future directions for our research. While both technological and furniture solutions were developed for BlueSpace, this article will focus primarily on the former ${ }^{\mathrm{i}}$.

\section{Workplace Issues}

Current workplace issues were examined through a series of interviews and focus groups with people from a variety of function areas (e.g. marketing, development) and a range of company types (from Fortune 100 corporations to dot-com startups).

\subsection{Interview Findings}

In total, 50 people were interviewed from six companies in either a small group or private setting. The interviews were focused on understanding individual work environments and consisted of a series of open-ended questions, (e.g. "what do you like best about your current office space?") as well as follow-up probes to elucidate particular points that were raised by the interviewees.

One of the constraints commonly mentioned by cubicle occupants is the need to seek out conference rooms even when the collaborative work involves only one or two other people. Since conference rooms are often a scarce commodity and meeting in them requires advance planning to reserve the space, spontaneous work groups can become frustrated, wandering the halls in search of an empty room to meet in. The preference is to continue the collaborative work in the space where it had its genesis, but "two people working around a screen is difficult, with three people it becomes impossible." In addition to collaborative work, other reasons mentioned for leaving the cubicle included managers 
seeking privacy to make a call or conduct a one-on-one review, and workers seeking to prevent disruption to surrounding cubicles when using a speaker phone.

Another concern users have is the need to "find a quiet space" to do heads-down work without interruptions and distractions. While some went to an enclave (i.e. a one-person conference room), most felt compelled to do their quiet work either at home or in their workspace during off-hours (either late at night or early in the morning). The major drawback to going to an enclave is that users invariably found that they have forgotten to bring something they need to get the task done. Many commented they are reluctant to leave their primary workspace since they usually do not have a network connection in the alternate workspace and they worry about being out-of-touch with their email and voicemail. Thus, user interviews clearly showed that task demands (e.g. need for quiet or privacy) occasionally drive cubicle occupants out in search of other spaces (home, enclave, conference room) because the task cannot be accomplished within the confines of the individual workspace. A representative user comment was: "most of the time that I spend outside of my cubicle is because the work that I do outside of that cubicle can not be performed in the cubicle."

An overriding user goal when trying to accomplish work that requires concentration is to minimize the number of "drive-by interruptions." These are instances where a colleague is walking by and remembers when he sees you that there is something he wants to ask you, or just stops in to inquire about your weekend. There is a clear need for "privacy on demand" which emerged from the interviews. This represents the ability to have the space be as open as necessary to support group interactions, as well as the ability to close the space up so that both visual and auditory privacy are possible. Users were 
seeking the technology equivalent of a "cone of silence", which could descend upon their workspace and prevent unwanted interruptions.

Lastly, users requested greater individual control over environmental issues that impact personal comfort. While most have adapted their dress code to deal with too hot/too cold issues (some wear a sweater, others opt for short sleeved T-shirts) it is perhaps the need to adapt that chafes more than the outcome. Many mentioned wishing for the "comforts of home" in a setting where a greater number of waking hours are spent than at home.

Following an analysis and review of the initial interviews, a storyboard was created with sketches of what the prototype solution might look like. The sketches were associated with narrative text (see Figure 1) describing the actions of the office occupant at various times throughout the day, along with a description of how the prototype helped the user deal with (or avoid) workplace issues as they came up. The next step was to create a series of threedimensional models showing various possible solution implementations (see Figure 2). These models, as well as iterations and variations of them, were discussed among team members. When we had arrived at a set of features and options that were the leading candidates, we took these forward to garner additional feedback from users. This feedback took place in the form of focus groups.

(FIGURE 1 GOES HERE OR NEAR)

(FIGURE 2 GOES HERE OR NEAR)

\subsection{Focus Group Findings}

The focus groups were conducted at two of the six sites that had participated in the interviews and observations phase. One of the dot.com companies and one of the large 
enterprises were selected for the additional feedback sessions. Each company had 10 employees participate in the focus groups, with a range of workers being represented (e.g., managers and non-managers, marketing and development). The framework for the focus group discussions consisted of: introductions, a high level commentary from each participant of the benefits and drawbacks of their current work environment, an introduction by the moderator of the model and proposed solution, feedback on the model, a brainstorming of necessary features and functions, ranking of features and a discussion of the value proposition. The resulting prioritized feature set is shown in Table 1.

\section{(TABLE 1 GOES HERE OR NEAR)}

The ranking of the features for Table 1 was based on their average score across both sessions, with a score of five being the highest possible value. When two features tied for the same score they are listed on the same line, separated by a slash. The participants were asked in particular to comment on several aspects of the model including:

o The Office Front panel (a small flat screen monitor) at the entry of the workspace that can be used to communicate with people who stop by the workspace when the occupant is away;

o The two large monitors on articulating arms. The first to be used as the primary display, the other as an information panel. The information panel can serve as a collaborative display when working with a colleague, or it can be used to monitor information such as one's calendar or stock prices while working in other applications on the primary display;

o The use of badges and badge readers that would detect the user's presence in the workspace and identify personalized preferences. 
o The light fixed above the office entry that is used to communicate availability status to colleagues;

o The use of wall openings (windows) for interacting with collocated colleagues or large groups;

The top ranked feature by the focus groups was the presence of multiple flat panel displays in the model. Users liked the additional flexibility, the ability to track multiple sources of information simultaneously, and the high degree of personalization that these would afford them. The use of Smart (or Active) Badges (Bokun, Krzysztof, 1998) was also seen as a key feature. A smart badge allows the office occupant to be identified and thus "recognized" by a badge reader in the space. In particular, focus group participants liked the ability to determine if co-workers are currently in their workspace, and the ability to have one's personalized environmental settings automatically triggered when entering one's space. They envisioned using the badge to populate information on the Office Front panel in a hoteling environment (i.e. who is sitting in the office today) as well detecting when conference rooms are not currently occupied, in order to return them to the list of available rooms.

While we will not go through a detailed discussion of each of the other features in the list, the last one we will mention is the Nap Space. This was first introduced as a necessary addition to the model during a discussion with participants from a dot.com company. It seemed fitting to the 16-hour workdays of some startup companies, but we were surprised when other participants in a different setting also added it to their prioritized list. The Nap Space was described by the participants as "something like a hammock or a folded up futon" in the personal workspace where office occupants could catch a quick catnap. 
While there were some clear winners in the feature list (the first three), the ranking of the features beyond the top few got somewhat diluted since the participants were voting on a long list of features that the group had created through brainstorming. Thus the last feature (the use of speech recognition) was included in order to list 10 features, but it did not garner a majority of votes across sites.

\section{BlueSpace}

Figure 3 shows the current BlueSpace implementation. The workspace currently occupies a 9' by 10' footprint and incorporates a set of sensors (too small to be seen in the photograph), for measuring ambient light, temperature, humidity, and noise level. Additionally, the desk chair is equipped with a pressure sensor connected to a wireless micro-controller, which detects if a person is sitting in the chair. An active badge and reader are used for presence detection and identification. A set of environmental effectors has been integrated into BlueSpace including various illuminating and signaling lights, as well as a Personal Environmental Module (PEM) (Antonelli, 2001). The PEM is composed of a fan system and a heat panel, designed to provide adjustable heat and airflow to desks in open-plan offices. The module is installed at the bottom of the main worktable.

\section{(FIGURE 3 GOES HERE OR NEAR)}

There are three flat panel displays in the space. The first, the Office-Front, is integrated into the Threshold component and is used for sharing information about the occupant such as his/her name, interests and current availability for interruptions. The two other displays (the Primary and Information Panel) are mounted on articulating arms. The Primary is for 
focused individual work and the second can be used for peripheral information monitoring, or collaboration. In addition to the three monitors, there is a steerable projection system we call the Everywhere Display projector (ED-projector) (Pinhanez, 2001). It uses a computer-controlled mirror to direct projected images onto virtually any surface in the workspace. The images are corrected for oblique projection distortion. . The ED-projector allows collaboration without the "two people working on the same screen" problem since any wall or tabletop can be transformed into a display.

\subsection{The Privacy/Collaboration Continuum}

One of the primary objectives in BlueSpace is to support users' need for privacy when performing tasks that require concentration or discretion, as well as their need to occasionally work collaboratively with colleagues. These needs can be viewed as opposite ends of the same continuum and require a set of protocols and tools to flexibly manage them throughout a workday. The cornerstone of our approach to privacy is the management and awareness of an individual's availability.

An individual can set his availability status (e.g. At Lunch, Please Do Not Disturb) by touching a tile on the Information Panel. The active badge system also automatically detects when the occupant enters or leaves the workspace, and changes the user's status to reflect whether he or she is actually in the workspace at the moment. The user can optionally write an informative message that elaborates upon the status. For example, "Be back" may be accompanied by the message "I'm in Hawthorne, back at 3:00pm." The availability information is displayed on the Office Front panel as well as by the colored status light. The status light is incorporated into the Threshold and thus shows the individual's availability from a distance (Free $=$ green, Busy=red or Gone=blue). In Figure 3 
the status light is green, indicating that the occupant is in the workspace and available to colleagues. The goal is to reduce "drive-by" interruptions by making people aware, before they stop at the entrance to the cubicle, that the occupant is busy and does not wish to be disturbed. In addition to displaying the availability information on the Office Front panel and through the status light, more detailed information about colleagues is shown through an awareness application that we call myTeam.

The myTeam application runs on the Information Panel. While not a true media space (Mantei, Baecker, Sellen, Buxton, Milligan, Wellman, 1991) in that it does not make use of any video images, it allows users to maintain a level of "general awareness" (Gaver, Moran, MacLean, Lovstrand, Dourish, Carter, Buxton, 1992) of co-workers by viewing their availability at-a-glance. Figure 4 shows an early implementation of myTeam towards the bottom of the Information Panel. Team members are represented by an icon (in later versions by a photograph), which reflects their current state (e.g. gone, busy, or available/free). Colleagues' presence or absence in their office is reflected by the presence or absence of their image in the application. The active badge and badge reader are used to automatically update a user's presence in myTeam. Thus, a user is not required to remember to change her status every time she steps in or out of the office.

\section{(FIGURE 4 GOES HERE OR NEAR)}

Allen (1984) in a seminal study on the impact of distance on probability of communication states that people need visual reminders of the presence of others, and that if people do not see each other, they will not have the opportunity for creativity-inducing contacts. Our hope with the myTeam application is to create a sense of social presence of fellow team members - the images of colleagues communicating not only status but also 
acting as visual reminders of the presence of co-workers who may be located on a different floor, building or continent. MyTeam allows users to register themselves as "Waiting" for a colleague that is currently in Do Not Disturb mode. Simply touching the icon of the busy individual registers a colleague to be notified when the busy or unavailable (e.g. gone) coworker becomes available. This has some similarities to Greenberg's Peepholes (1996) system where a user could "ambush" somebody upon their return to the office by having the system notify him when the individual returned. MyTeam displays the identity of any individual who is waiting. This way, a worker can quickly check whether there are people waiting to work with her, and try to make herself available.

Two major furniture components help the office occupant easily reconfigure the workspace between an area that supports small group collaboration, and one that facilitates privacy and heads-down work. These are the Monitor Rail and the workspace Threshold. The monitor rail is a moving rail that travels the width of the workspace with the dual monitor arms providing 240 degrees of freedom, allowing the user to position the screens and work anywhere in the area. The Threshold, is also moveable along the width of the workspace and provides both visual and territorial privacy to the user.

BlueSpace also monitors the presence of other active badges in the workspace to automatically reconfigure what information is visible if a visitor is detected. When a visitor enters the space, any confidential or private information currently displayed either by the ED-projector or on the Information Panel (e.g. email, calendar entries) is immediately hidden. The personal image displayed with the ED-projector can also be toggled to a more "public" image.

\subsection{Workspace Personalization}


BlueSpace gives users the ability to personalize the environmental settings in the workspace, thus contributing to an increased sense of control over their environment. The Information Panel provides touch-screen computerized control of the lighting and temperature in the workspace, empowering the occupant to configure the settings based on the current task, as well as how she feels at the moment. Additionally, the space can sense and automatically adjust the environment to meet a personal profile setting linked to the identification provided by the active badge. The PEM provides fine-tuning of local temperature by allowing for adjustments to both temperature and speed of airflow, as well as radiant heat. The fluorescent ceiling lights above the space can be boosted or dimmed and the task lights embedded into the movable threshold can be adjusted for color as well as intensity. This allows the occupant to simulate a broad range of lighting effects ranging from the warm appearance of natural sunlight to cold florescent lights.

The environmental controls are displayed on the Information Panel (Figure 4) but could run on the Primary display instead. The interface was designed to provide one touch control of frequently used applications as well as information at a glance. The current implementation shows sensor outputs such as the office temperature, humidity, ambient light and noise levels. Additionally it includes the occupant's calendar entries for the day, a to-do list, a collaborative awareness application (called myTeam), webcam images, the weather and outside temperature. The intention of the Information Panel is that these items would be individually configured to display information that is frequently referred to by the occupant. 
The Office Front display, allows the user to add a personal touch to his workspace that can be viewed by people walking by. This display can be customized to include favorite images as well as text describing work activities and other interests. The ED-projector can be used to project a favorite image whenever it is not being employed by the user as an additional computer display. In Figure 3 the ED-projector is displaying the image of an outside window. This can be connected to a webcam, thus giving every office the potential to be a "window office". The ED-projector can alternate between displaying personal photographs, outside views, pleasing images (e.g. artwork) or notifications (e.g. arrival of urgent email) depending on the context and the user configurations.

BlueSpace uses context awareness to help manage the workspace. Upon arrival in the morning, the active badge system detects the presence and identity of the individual entering the empty office. The system determines if the occupant is the workspace owner, and if so, looks up her preferences for lighting, temperature, decoration, etc. and automatically configures the workspace to these preferences. Conversely at the end of the day, when the occupant changes his status to reflect his departure for the day, the workspace is automatically reconfigured to its preset nighttime settings (e.g. lights are turned off, computer is locked, temperature turned down). We also use awareness of where the occupant is located, (e.g. sitting in chair) to direct where to project the notifications (e.g. summary of urgent email messages). The intent is to enable a worker to concentrate on the work at hand, but still allow notification of important events. When the occupant has set his status to Do Not Disturb he is only notified of urgent email (although this can userconfigured). Urgent email notification consists of only the sender, subject and a summary of the message. Figure 5 illustrates a user glancing at a notification on the wall. 
(FIGURE 5 GOES HERE OR NEAR)

We are exploring the use of a noise level detection sensor to help avoid situations where the occupant's behavior disrupts the work of nearby offices. Our basic approach is to use a mechanism that warns the worker through visual feedback if noise levels exceed a predetermined threshold. We are experimenting in parallel with use of a noise canceling mechanism that is part of the PEM unit.

\section{User Responses}

Since BlueSpace was completed in January 2002, we have had the opportunity to show the workspace to over 800 people. While most of these have been demonstrations lasting about 30 minutes, in many cases the visitors remained for over an hour after the demonstration to discuss their impressions and give us feedback. Clearly, the issues of privacy, concentration and personalization that BlueSpace attempts to address, resonated strongly with the visitors. Many of the people viewing the workspace could relate keenly to the problems of working in cubicles, and almost all wanted to have a BlueSpace installed for them at their workplace.

The element that usually causes the greatest stir and reaction is the Everywhere Display projector, especially when the capabilities for making any surface interactive by combining the ED-projector with computer vision are discussed. Interestingly enough though, when visitors are queried which of all the BlueSpace components could they live without, if they had to forgo something, the ED-projector is the element most frequently mentioned. Sometimes it is seen as overkill for an individual office, and users suggest that it would be most useful if placed in a commons area shared by multiple BlueSpace units. 
In general, people react most favorably to the ability to regain a sense of control of their environment, and the ability to personalize the space. Visitors also connect strongly with the awareness and communication features of the myTeam software, mentioning that while they want to avoid unwanted interruptions, they are also looking for cues when to initiate interaction with team members. "We have all played telephone tag with co-workers, or been frustrated to walk over to a colleague's cube, only to find that he has temporarily stepped away" is a representative comment of the current state of team communications.

Many visitors wondered out loud whether employees at their company would actually respect the privacy signals, and some suggested that at their workplace everyone would immediately go into Do Not Disturb mode and stay that way permanently. Visitors from the Facilities and Real Estate branches of corporations mentioned that they would want to see some evidence of increased productivity, greater worker satisfaction or higher retention as a result of installing a BlueSpace environment, especially if BlueSpace were to entail any spending above the existing workspace budget. To answer these questions and more, we are proceeding with a Field Trial phase for BlueSpace. Multiple BlueSpaces will be installed at different companies for a period of several months and user responses will be measured and recorded. We would like to understand the impact that BlueSpaces have on workplace dynamics, the social protocols that emerge from use of status indicators and awareness information, and whether "drive-by interruptions" are significantly reduced.

\section{Discussion and Future Work}

BlueSpace is a first iteration in a research agenda that is examining the impact of "smart" spaces: environments where the digital and physical worlds converge. We are 
interested in developing spaces that address everyday user needs, where sensors and actuators assist in the management of the environment, and where the interaction between the user and environment is seamless.

New interaction paradigms that take advantage of off-the-desktop display devices such as the ED-projector support the move away from a desktop-centric approach to addressing computing needs. The goal is to support user interaction through simple hand gestures such as pointing and touching, detected by a camera. Several of the challenges that need to be overcome to reach this goal include dynamically estimating surface characteristics and reliably capturing user input by means of analyzing hand motions on or near the surface.

Another area that we would like to investigate further is communication between the occupant and the space. Currently, all interaction happens through the Information panel. We are exploring the idea of using an avatar for the space: a personified manifestation of the software that assists in the management of the workspace when the occupant is present or absent. Manifestations such as these assist from a speech recognition point of view since often the user will turn towards the avatar to address it, facilitating directional microphone capture of the user input. Also, if speech commands are used for control of office appliances and environmental settings, the avatar provides a conversational partner for the user, thus eliminating the problem presented when users are required to "speak to the walls." The avatar could also play a social role, greeting visitors and taking messages when the office occupant is absent from his space.

On a longer-term horizon, we would like to explore the relationship between mobile computing and smart environments. Mobile devices have increasingly become an integral part of the workspace computing. Providing infrastructure services to mobile devices while 
preserving the integrity of the private, virtual space remains a challenging issue. We plan to extend the BlueSpace components with service discovery, security, and gateway services, as well as applications to facilitate remote collaboration and telepresence.

\section{Acknowledgements}

The authors would like to thank all the BlueSpace team members that worked towards making the vision a reality: Paul Moskowitz, Mark Podlaseck, Mark Schunzel, Danny Wong, and Sachiko Yoshihama. We would also like to thank our colleagues at Steelcase, Joe Branc, Charlie Forslund, Jason Heredia, Mark Baloga, Joel Stanfield and the others, whose dedication, hard work and creativity made it all possible.

\section{References}

[1] ALLEN, T.J. (1984) Managing the Flow of Technology: Technology Transfer and the Dissemination of Technological Information within the R\&D Organization. Cambridge, MIT Press.

[2] ANTONELLI, P. (2001) Workspheres: Design and Contemporary Work Styles. The Museum of Modern Art, New York, New York, NY.

[3] BAUMAN, F. S., ARENS, E. (1996) Task/Ambient Conditioning Systems: Engineering and Application Guidelines. Center for Environmental Design Reseach, UC Berkeley, publication number: CEDR-13-96.

[4] BOKUN, I., KRZYSZTOF, Z. (1998) Active Badges - The Next Generation. In Linux Journal, October

[5] BUDD, C. (2001) The Office: 1950 to the Present. In Workspheres: Design and Contemporary Work Styles, P. ANTONELLI (ed.). The Museum of Modern Art, New York, New York, NY. pp. 26-35.

[6] FISK, W. J., ROSENFELD, A. (1997) Estimates of Improved Productivity and Health form Better Indoor Environments. Indoor Air, 7: 158-172.

[7] GAVER, B., MORAN, T., MACLEAN, A., LOVSTRAND, L., DOURISH, P., CARTER, K., BUXTON, W. (1992) Realizing a Video Enviroment: EuroPARC's Rave System. In Proceedings of the CHI'92 Conference on Human Factors in Computing Systems.

[8] GREENBERG, S., (1996) Peepholes. In Proceedings of the CHI '96 Conference Companion on Human factors in Computing Systems

[9] MANTEI, M., BAECKER, R., SELlEN, A., BUXTON, W., MILliGAN, T., WELLMAN, B. (1991) Experiences in the Use of a Media Space, in Proceedings of the CHI '91Conference on Human Factors in Computing Systems.

[10] MENDELL, M.. (1993) Non-specific Symptoms in Office Workers: A Review and Summary of the Epidemiologic Literature, Indoor Air, 3: 227-236. 
[11] NARDI, B., WHITTAKER, S., BRADNER, E. (2000) Interaction and Outeraction: Instant Messaging in Action. In Proceedings of the CSCW '00 Conference on Computer Supported Collaborative Work.

[12] O'CONAILL, B., FROHLICH, D. (1995) Timespace in the Workplace: Dealing with Interruptions, In Proceedings of the CHI '95 Conference on Human Factors in Computing Systems.

[13] PINHANEZ, C. (2001) The Everywhere Displays Projector: A Device to Create Ubiquitous Graphical Interfaces. in Proceedings of Ubiquitous Computing 2001 (Ubicomp'01). Atlanta, Georgia.

[14] TEASLEY, S., COVI, L., KRISHNAN, M., OLSON, J. (2000) How does Radical Collocation Help a Team Succeed. In Proceedings of the ACM 2000 Conference on Computer Supported Collaborative Work. 


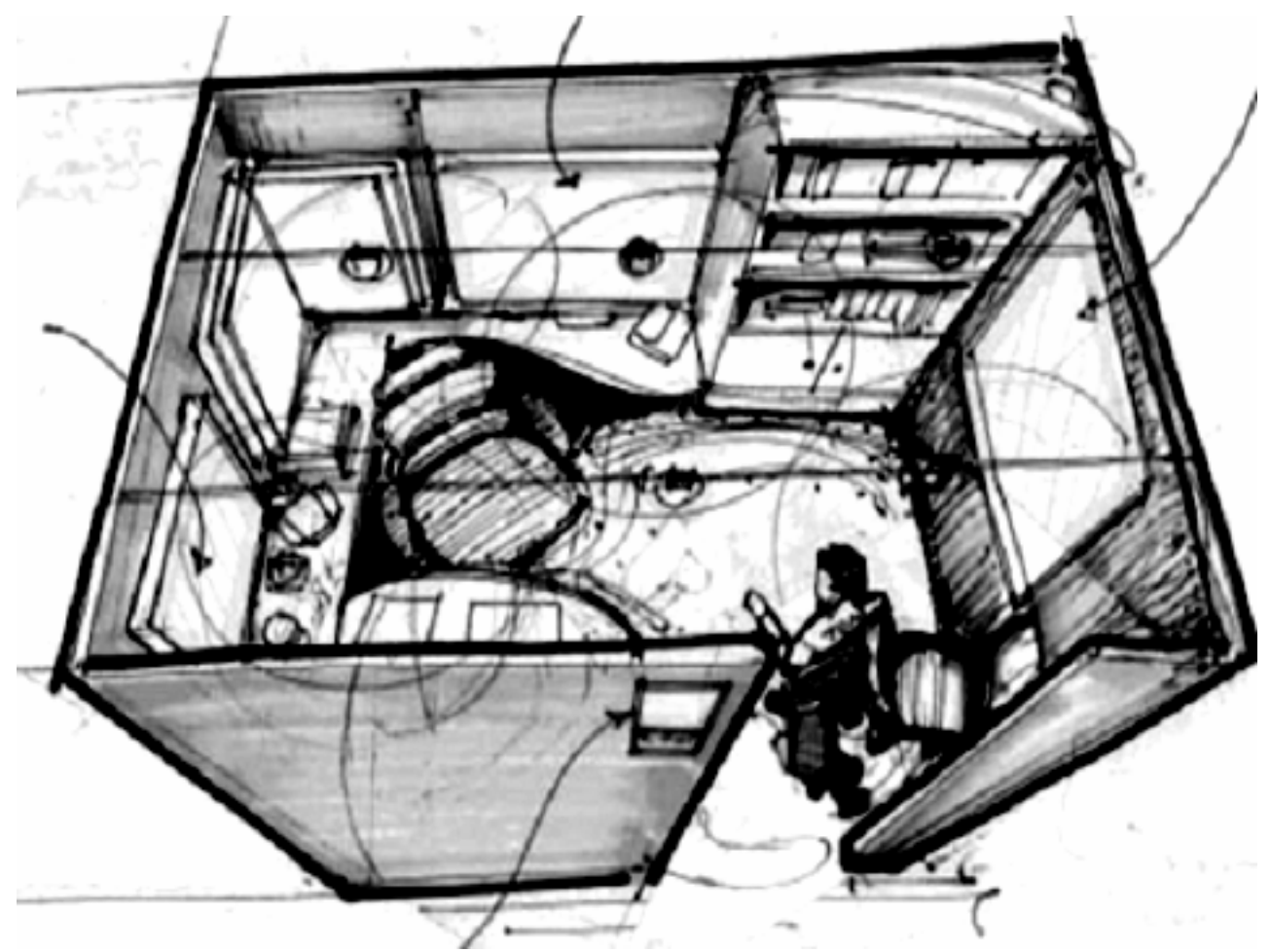

Tom is a busy account executive at a creative ad agency. As he enters his personalized, adaptive workspace for the first time that morning he is automatically recognized. Several things happen to herald the beginning of a new productive workday for Tom. The Information Panel, which he uses to stay in touch with his ever changing world, is activated and comes up with his calendar immediately visible without Tom needing to logon. His preferences for light and temperature are known and his personalized BlueSpace automatically moves to those settings.

Figure 1. An image from the BlueSpace storyboard 


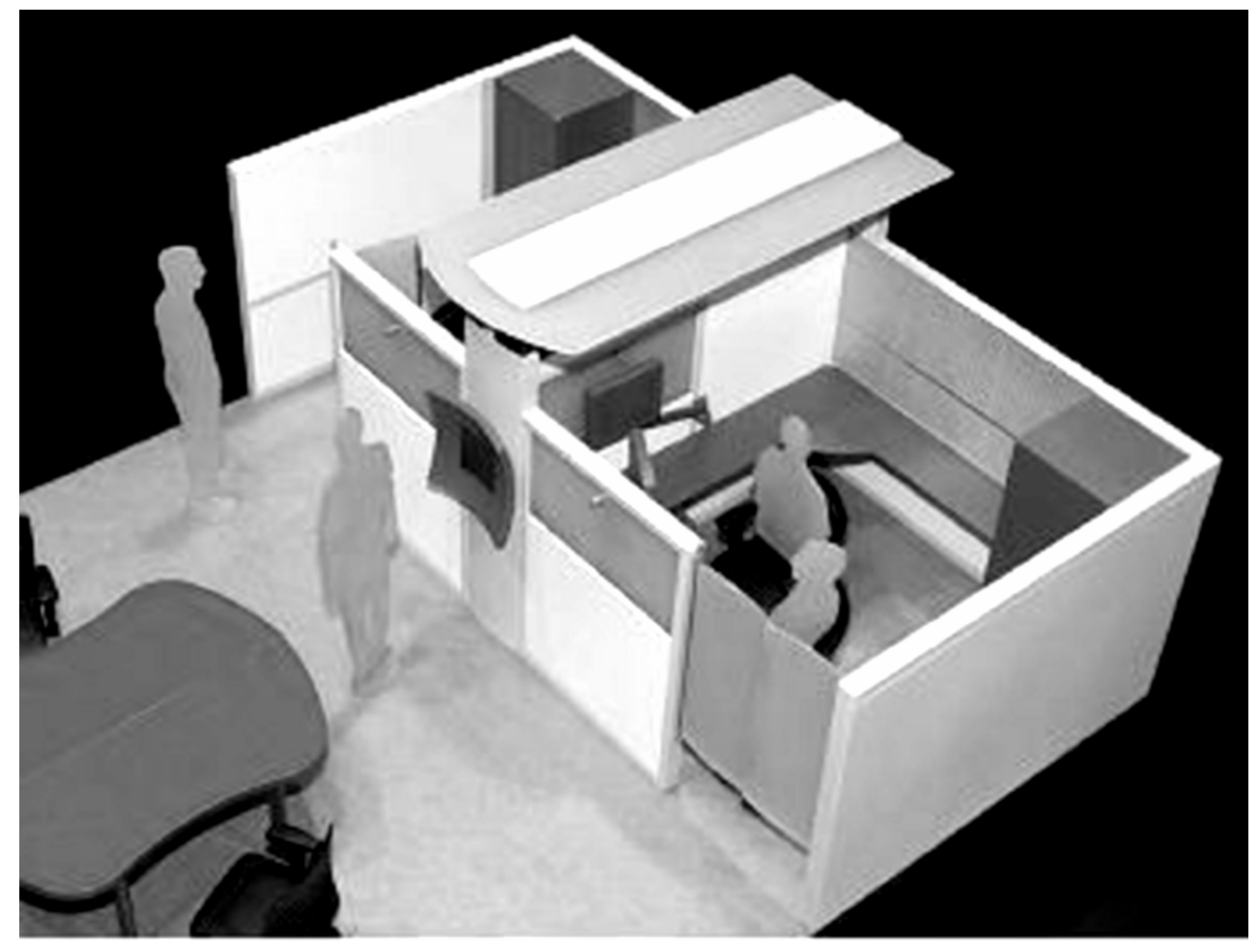

Figure 2. An early BlueSpace model image. 


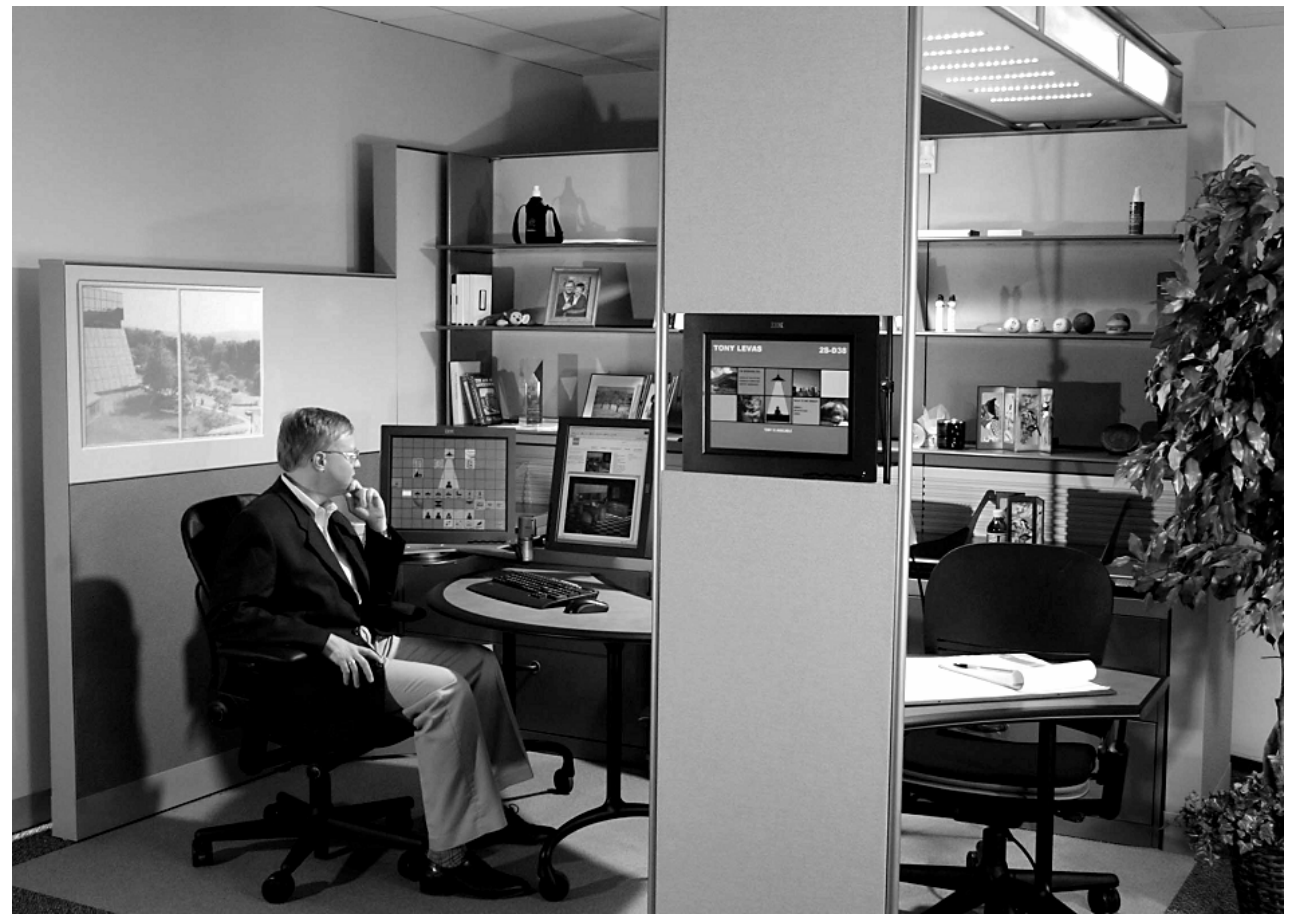

Figure 3. The BlueSpace prototype workspace. 


\begin{tabular}{|l|l|}
\hline FEATURE & RANKING \\
\hline Multiple displays & 3.20 \\
\hline Smart badges for identification & 3.00 \\
\hline Privacy on demand (both visual and audio) & 3.03 \\
\hline Aesthetically pleasing workspace & 2.23 \\
\hline Access to open space and common areas & 2.00 \\
\hline Digital recording device in conference rooms & 1.83 \\
\hline Support for wireless & 1.83 \\
\hline Individual control over lighting & 1.50 \\
\hline Collaborative space / Nap space & 1.33 \\
\hline Use of Speech Technology & 0.70 \\
\hline
\end{tabular}

Table 1: Top ten user requirements from focus group discussions 


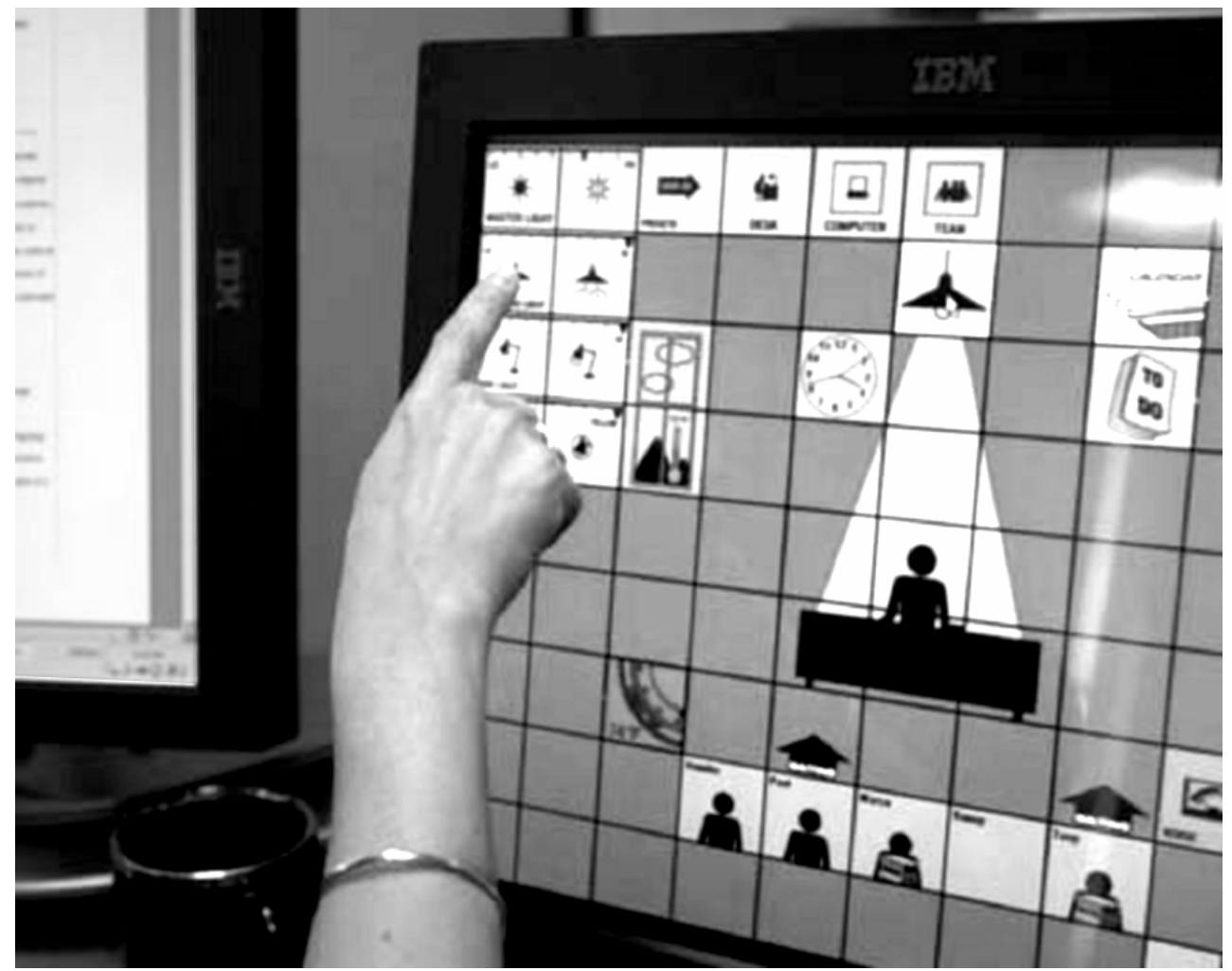

Figure 4. The touch screen Information Panel 


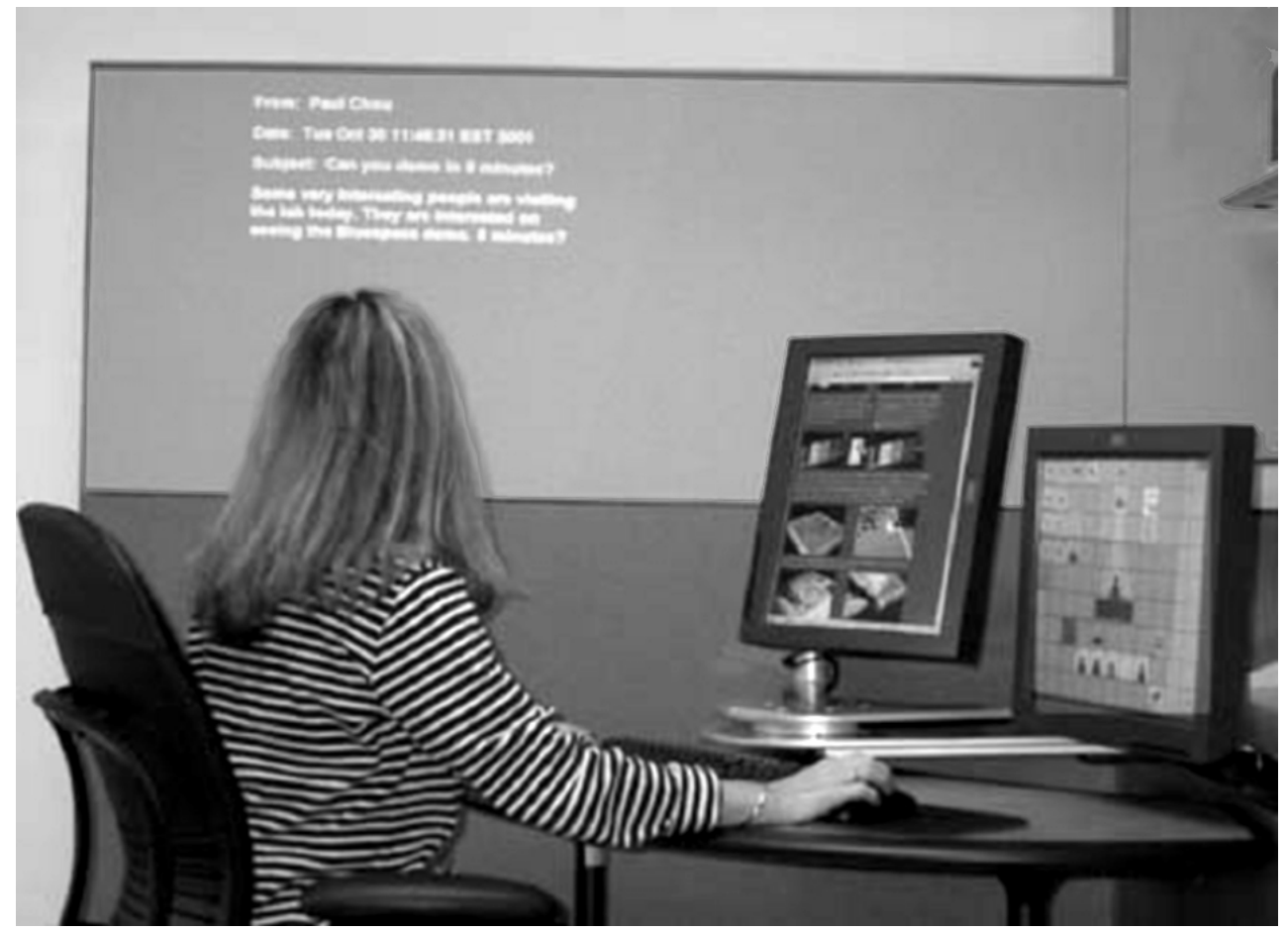

Figure 5. Peripheral notification of email using the Everywhere Display

i The BlueSpace prototype was developed in cooperation between the research department at Steelcase, a large furniture company, and IBM Research. 